https://helda.helsinki.fi

\title{
Impure placebo is a useless concept
}

\section{Louhiala, Pekka}

2015-08

Louhiala , P , Hemilä , H \& Puustinen , R 2015 , ' Impure placebo is a useless concept ' ,

Theoretical Medicine and Bioethics , vol. 36 , no. 4 , pp. 279-289 . https://doi.org/10.1007/s11017-015-9336-6

http://hdl.handle.net/10138/228057

https://doi.org/10.1007/s11017-015-9336-6

unspecified

acceptedVersion

Downloaded from Helda, University of Helsinki institutional repository.

This is an electronic reprint of the original article.

This reprint may differ from the original in pagination and typographic detail.

Please cite the original version. 


\title{
Impure placebo is a useless concept
}

\author{
Pekka Louhiala1, Harri Hemila1, Raimo Puustinen ${ }^{2}$ \\ ${ }^{1}$ Department of Public Health, University of Helsinki, P.O. Box 41, 00014 Helsinki, Finland \\ ${ }^{2}$ School of Medicine, University of Tampere, 33014 Tampere, Finland
}

This is a manuscript version of a paper published in its final version as: Louhiala P, Hemilä H, Puustinen R.

Impure placebo is a useless concept.

Theoretical Medicine and Bioethics 2015;36(4);279-289.

http://www.ncbi.nlm.nih.gov/pubmed/26215744

http://dx.doi.org/10.1007/s11017-015-9336-6

\begin{abstract}
Placebos are allegedly used to a wide extent in general practice. Surveys reporting high level usage have, however, combined two categories, "pure” and "impure" placebos. The wide use of placebos is explained by the high level usage of “impure placebos". In contrast, the prevalence of the use of "pure placebos" has been low. Traditional "pure placebos” are clinically ineffective treatments, whereas "impure placebos" form an ambiguous group of diverse treatments that are not always ineffective. In this paper, we focus on the concept "impure placebo" and demonstrate problems related to it. We also show that the common examples of "impure placebos" are unsound or absurd from the point of view of clinical practice. We conclude that “impure placebo" is a useless concept and should not be used in scientific or medical literature.
\end{abstract}


According to a recent survey, almost all British general practitioners (GP's) use placebos in their practice [1]. This result was widely reported by news agencies [2, 3]. The high prevalence of placebo use was, however, composed primarily of the use of so called "impure placebos", which is a new concept in placebo research. According to the survey, 97\% of the GP's had used “impure placebos” at least once during their career, whereas only 12\% had used "pure placebos" which are the traditional ineffective treatments (such as lactose pills). Because it was the "impure placebo" that had the high level usage by GPs, the meaning of the concept should be properly considered.

\section{Background}

Much of the confusion related to the concepts "placebo" and "placebo effect" is due to their many meanings and ambiguous nature. Three decades ago, Grünbaum [4] wrote that “... the medical and psychiatric literature on placebos and their effects is conceptually bewildering, to the point of being a veritable Tower of Babel.” The situation is not much better today, and, in spite of the growing academic interest in the nature of placebo effects, there is no consensus about the definitions of these terms.

This confusion is still prevailing. The basic paradox with the term "placebo effect” is that, by definition, a placebo - an inert substance or procedure - cannot elicit an effect [5, 6]. A further problem arises if "placebo" is understood as an upper level category that is divided into two lower level categories, “pure” and "impure” placebos [7, 8]. In several surveys these two categories were taken for granted; yet their problematic nature has also been acknowledged:

\footnotetext{
"What is considered to be an impure placebo varies considerably among studies and it is unclear and subjective when an intervention is a placebo or an active or effective intervention. Surveys investigating definitional aspects reveal considerable disagreement regarding whether defined interventions should be considered (impure) placebos or not .... With this lack of clarity it is doubtful whether the evaluation of something such as 'the prevalence of the use of impure placebos' makes sense.” [9]
}

Fässler et al. [9] concluded by noting that "The academic concept of an impure placebo might inappropriately reflect the complex situations and motivations in which health care professionals apply interventions which are not backed up by scientific evidence.”

Acknowledging the problems in using the concept "impure placebo" did not, however, prevent the same authors from using that notion in their subsequent article "Widespread Use of Pure and Impure Placebo Interventions by GPs in Germany” [8].

In this paper we argue that the concept “impure placebo" is not just scientifically unsound but that it 
may also be actively harmful. It causes confusion for both the scientific community and the general public, in particular because the term "placebo" carries a negative connotation in the context of clinical medicine. In our view, the concept “impure placebo" has no relevant use in medical literature.

\section{Impure placebo}

The earliest division of the concept "placebo" into the subconcepts "pure” and "impure” placebo we were able to trace was in the second volume of the Cornell Conferences on Therapy in the 1940s, which included a lengthy discussion titled "The Use of Placebos in Therapy” [10]. In that discussion, Dr Eugene DuBois divided placebos into three classes. The first was pure placebos, the traditional bread pills or lactose tablets with no significant physiological effects. The second was impure placebos that were "adulterated with a drug that might have some pharmacological action, such as tincture of gentian or a very small dose of nux vomica. That is the adulterated placebo, the false placebo, the bastard placebo, you might call it” [10]. The third group of placebos in DuBois' classification was “the universal pleasing element which accompanies every prescription.”

Traditionally, DeBois' first and third classes have been the source of the confusion about placebos [11, 12]. The first definition refers to the concept in the context of controlled trials. The third refers to practical aspects of good doctoring, i.e. the "placebo effect" generated by physicians. Our focus in this paper is the second class, currently termed the "impure placebo".

After the Gold et al. paper, the impure placebo concept has been occasionally mentioned in the medical literature $[13,14]$. Nevertheless, the concept has remained unfamiliar in wider scientific circles. Recently, the impure placebo concept was used in surveys on how extensively physicians use "placebos" [1, 7, 8, 15-17] and this attention has made the concept currently important.

To illustrate the problems of the "impure placebo", we first analyse three definitions of the concept, as presented in some articles. We then discuss examples of impure placebos that are explicitly mentioned in the surveys.

\section{Definitions of impure placebo}

Definitions for "impure placebo" were given in all of the surveys mentioned above. These definitions overlap significantly and therefore we describe only three definitions from studies addressing Swiss general practitioners and paediatricians [7], Swiss general practitioners [16] and British general practitioners [1].

Fässler et al. [7] defined impure placebos as "substances or methods which do have a known pharmacological or physical activity but which cannot be expected to have any direct therapeutic effects for the respective disease and in the chosen dosage.” [our italics] 
Fent et al. [16] considered impure placebos to have pharmacological effects, "but the effect on the specific disease the substance is prescribed for has not been proven or is uncertain.” [our italics]

Howick et al. [1] stated that impure placebos are "substances, interventions or 'therapeutic' methods which have known pharmacological, clinical or physical value for some ailments but lack specific therapeutic effects or value for the condition for which they have been prescribed.” [our italics]

There are several problems in these definitions:

Firstly, it is important to distinguish between the following two questions: 1) does an intervention have an effect? and 2) what is the mechanism of the effect? This distinction is not made in the above definitions. There are effective interventions for which we do not know the mechanism, yet they are effective. Similarly, there are known mechanisms that do not correspond to clinically relevant effects.

Terms such as "specific" and "direct” are commonly used in the placebo literature, but their meaning is hardly ever explained or discussed. If an intervention does not have a "specific” or "direct” effect, referring to a "nonspecific" or "indirect" effect is just another way of saying that we do not yet know the mechanisms of the intervention. However, this does not mean that the intervention is ineffective.

Furthermore, especially within the field of general practice, in which the surveys on impure placebo have mainly been carried out, the symptoms and worries that the patients present to their physicians are often nonspecific in the medical sense. Often there are no specific treatments in the same sense as for specialist care with more severe and well-defined diseases. We do not see any justification for labelling the alleviation of patients' symptoms and worries as impure placebos.

Secondly, if the effect of a treatment "has not been proven", it does not imply that there is no effect. “Absence of evidence is not evidence of absence” was the message of an important paper in the BMJ [18]. Smoking, for example, was harmful before the harm had been undisputedly proven in large cohort studies. Furthermore, "has not been proven" is a question which is often limited by resources. Pharmaceutical companies have extensive resources for randomised controlled trials to test their own patented drugs, but similar resources are not available for most non-patentable treatments. There is no basis for the belief that all untested treatments are ineffective.

Thirdly, the meaning of "uncertain" is not clear in the definition by Fent et al. [16]. They may, for example, refer to lack of consensus in the medical community. In this meaning "certainty" and "uncertainty" do not represent a dichotomy but refer to extreme points on a continuum. Furthermore, in clinical practice, many or most treatments are "uncertain” in the statistical sense. For example, if a treatment helps $50 \%$ of the participants in a randomised trial, the clinician does not know to which half his or her patient belongs, although it is reasonable to use the treatment. Average benefit does not guarantee that a treatment works for a particular patient.

Inconsistencies between definitions create a situation in which researchers do not measure the same 
phenomena. As Meissner et al. [8] wrote: “A major problem in any survey on placebo use is the concept of impure placebo. The decision whether an intervention is still an active treatment or a placebo depends on personal attitudes and situational factors.”

As long as there is no common agreement on how impure placebos can be defined unambiguously, the scientific value of surveys on its use is low. By analogy, if different psychiatrists would all have their own definitions for the concept “depression”, studies addressing the prevalence of depression in different countries could not be compared.

Table 1. Treatments labelled "impure placebos" in six surveys* on the use of placebos

\section{Treatments labelled as impure placebos in two or more surveys}

Antibiotics for suspected viral infections (1-6)

Non-essential physical examinations $(1,2)$

Non-essential technical examinations of the patient (blood tests, X-rays) $(1,2)$

Peppermint pills for pharyngitis $(1,2)$

Phytotherapeutics/herbal supplements $(3,6)$

Positive suggestions $(1,2)$

Sedatives $(3,4)$

Sub-clinical doses of otherwise effective therapies $(1,6)$

Vitamin infusions for cancer $(2,5)$

Vitamins without approved indications $(2,3,4,6)$

\section{Treatments labelled as impure placebos in only one survey}

Acupuncture (3)

Cognitive behavioural therapy (CBT)** (1)

Complementary and alternative medicine (CAM) whose effectiveness is not evidence-based (1)

Conventional medicine whose effectiveness is not evidence based (1)

Ferrous sulphate** (1)

Gesture and intonation in addition to positive suggestion** (1)

Homeopathic remedies (3)

Ibuprofen or other pain medication for symptoms unrelated to pain (6)

Joint injection** (1)

Massages (3)

Medication** (1)

Minerals and trace elements (3)

Nutritional supplements for conditions unlikely to benefit from this therapy

(such as vitamin C for cancer) (1)

Off-label uses of potentially effective therapies (1)

Physiotherapy** (1)

Probiotics for diarrhoea (1)

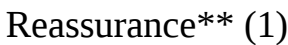

Referral to website** (1)

Simple ointments and/or bandages for contusions without visible skin damage (2)

Tell patient of own or my family member with the same problem* (1)

Unnecessary referrals** (1)

*Studies: 1. Howick et al., 2013; 2. Fässler et al., 2009; 3. Meissner et al., 2012; 4. Hróbjartsson and Norup, 2003; 5. Fent et al., 2011; 6. Kermen et al., 2010.

**Included in Howick et al. ${ }^{1}$ Table 2 category "Other" 


\section{Proposed examples of "impure placebos"}

Apart from the conceptual problems related to the definition of the term "impure placebo", there are also problems with classifying actual treatments into that category. In table 1, we have listed all treatments that have been labelled "impure placebos" in published surveys. All of the listed items do not appear in all of the articles, which is a feature reflecting the differences in the definitions. In this section we include only examples of “impure placebos” that are mentioned in at least two surveys:

Antibiotics for suspected viral infections. [1,7,8,15-17]. Most infections of the upper respiratory tract are viral in origin, and therefore antibiotics are not beneficial for most of these infections. However, in a clinical setting, it is usually not possible to be fully certain that a particular infection is not caused, at least partly, by bacteria. Kaiser et al. [19] found that antibiotics were clinically beneficial for a subgroup of patients with upper respiratory infections whose nasopharyngeal secretions contained Haemophilus influenzae, Moraxella catarrhalis, or Streptococcus pneumoniae. In practice, physicians prescribe antibiotics on the basis of the nature, severity, and duration of the symptoms of individual patients [20, 21]. It is not reasonable to classify all antibiotic use for the diffuse category of "suspected viral infections" as "impure placebo" treatment.

Non-essential physical examinations and non-essential technical examinations of a patient (blood tests, $X$-rays) [1, 7]. It is not possible to be certain which physical or technical examinations are diagnostically essential for an individual patient. All experienced physicians recall cases in which an examination that originally seemed non-essential unexpectedly revealed important findings and vice versa. There is no sharp line between obviously essential and obviously unsound examinations. Instead, there is a wide region in which the physician must make subjective decisions, and different physicians make different decisions. "Non-essential examinations" is not a reasonable category for impure placebos.

Peppermint pills for pharyngitis [1,7]. To use an analogy, pain killers do not kill viruses, and, in that respect, they do not influence the aetiology of viral pharyngitis. However, pain killers improve the quality of life of patients who have sore throats, and improved quality of life is one of the most important goals of medicine. Similarly, peppermint pills may alleviate the pain of a sore throat or cough, and there is a biological rationale for this effect. The pills increase the production of saliva, and the saliva increase may protect the mucosa of the pharynx from airflow and aid the drainage of mucus. We were not able to find randomised trials examining the effect of peppermint pills on sore throat, but the experience of numerous patients, including those of the authors of this paper, indicates that peppermint pills are useful in alleviating the symptoms of pharyngitis. It is unlikely that pharmaceutical companies will become interested in carrying out trials on peppermint pills, and it is also unlikely that public health authorities will prioritize funding for such trials. However, a lack of published trials does not imply that peppermint pills are ineffective in improving the quality of life of pharyngitis patients [18].

Phytotherapeutics/herbal supplements [8, 17]. This is a broad category, and it is not meaningful to pool 
all such treatments into a single group and classify them as "impure placebos" as was done in the two surveys. Although we are greatly sceptical of most treatments falling into this category, there is evidence that some phytotherapeutic preparations are effective. St. John's wort extracts, for example, have been beneficial in the treatment of mild to moderate depression in several placebo-controlled trials [22].

Positive suggestions [1,7]. Physicians often assure their patients with such statements as "You will certainly feel better in a few days” or "I'm sure this will help you”. Utterances such as these are an essential part of good doctoring in any clinical setting all over the world. It is not reasonable to argue that such assertions "cannot be expected to have any direct therapeutic effects for the respective disease and in the chosen dosage. To classify good doctor-patient relationships as impure placebos is absurd.

Sedatives $[8,15]$. This is a broad category that includes, for example, benzodiazepines. Sedatives are used for a large number of medical indications, and it is unreasonable to dichotomise their clinical use into "indicated use" and "impure placebo" on the basis that they either have or lack "specific therapeutic effects or value for the condition for which they have been prescribed”.

Sub-clinical doses of otherwise effective therapies [1, 17]. Individual patients respond to medication differently because they metabolise drugs differently. A subclinical dose for one patient may be clinically effective for another, and "normal” doses may be too low for some patients. Particularly in symptomatic treatment, it is common to start with a low dose and slowly increase the dose until the patient responds or has unwanted side effects [23]. The use of drugs in low doses cannot be labelled categorically as an “impure placebo".

Vitamin infusions for cancer [7, 16]. Using vitamin C to treat cancer patients is not just a question of whether a high concentration of vitamin C might kill cancer cells. There is evidence that a substantial proportion of hospital patients have a vitamin C deficiency [24, 25]. Therefore, vitamin C may be beneficial for some hospital patients with or without cancer [26, 27]. There are also relevant case reports of vitamin C infusions being used to treat cancer patients [28]. There is no basis to classify vitamin C treatments of cancer patients categorically as “impure placebos".

Vitamins without approved indications [7, 8, 15, 17]. Lack of vitamins was identified during the first part of the $20^{\text {th }}$ century as the explanation for diseases that we nowadays classify as deficiency diseases. For most vitamins the approved indication is to treat deficiency, but this does not mean that they cannot have other effects. For example, systematic reviews have shown that vitamin $\mathrm{C}$ shortens the duration of colds [29], reduces the decline in forced expiratory volume (FEV1) caused by exercise [30], and reduces blood pressure [31]. There is no basis to classify all use of vitamins for purposes other than treating deficiency diseases as an "impure placebo" even though the clinical role of vitamins in non-deficiency diseases is still a controversial issue.

The preceding examples show that "impure placebo" is not a reasonable scientific concept. From the 
point of view of clinical work, all nine examples are unsound or even absurd.

\section{Methodological issues in surveys on the use of impure placebos}

Surveys on impure placebos have queried primary care physicians on their use of such placebos by sending questionnaires to a number of practising physicians [1, 7, 8, 15-17]. We point out two methodological problems relevant in the surveys.

Firstly, it is not clear how the responding physicians interpreted the definitions of impure placebos when they considered the examples of impure placebos in the list of the questionnaires. Some physicians may have counted all cases in which they prescribed a substance or method on the list, while others may have counted only those cases in which they prescribed impure placebos to please the patient. Thus, there can be large variation in how the respondents interpreted the survey questions.

Secondly, it is not clear how the surveyed physicians interpreted "prescription" or "use" of impure placebos. At least the following interpretations are possible: 1) writing a formal prescription for an impure placebo without informing the patient that the physician believed the impure placebo to be pharmacologically ineffective in this particular case, 2) verbally suggesting that the patient might test a treatment the physician considered ineffective, and 3) allowing the patient to continue the use of alternative medicine treatments that the patient had initiated himself or herself although the physician considered them useless. These situations differ to an important degree, but they have not been addressed in the impure placebo surveys.

\section{The impure placebo concept may even be harmful}

The concept impure placebo is not just meaningless, it may also be harmful, for the following reasons.

Firstly, ambiguous concepts lead to ambiguous thinking. For example, the conclusion that "placebos are commonly used in UK primary care” [1] is meaningless because it was based on combining the use of both "pure” and "impure” placebos. Most readers assume that "placebo" in such a context refers to the “pure placebo" and it is unlikely that readers understand that physicians’ positive assertions, peppermint pills, sedatives, administering antibiotics to the common cold, etc. are counted as "placebos”.

Secondly, published conclusions distort the impression of medicine in the eyes of the general public. Media coverage of the study by Howick et al. included the following headlines: "Many UK doctors give useless drugs, treatments” [2] and "The placebo effect: doctors admit prescribing unproven treatments, unnecessary tests and pills with no active ingredient” [3]. Such secondary reporting gives to the general public a grossly misleading view of medical practice and may create unjustified mistrust among the public towards the medical profession. 
Thirdly, the concept "impure placebo" undermines the importance of the physician-patient relationship and the context of care. The emotions that are associated with the term "placebo" are negative, since placebos are commonly described with terms such as "dummy" or "sham" and the placebo effect with expressions such as "suggestion”. Therefore, terms including the word “placebo" carry such pejorative connotations regardless of what has been written about their importance, existence, and supposed mechanisms.

Fourthly, because the concept has no unambiguous definition, empirical research on the use of "impure placebos” is a waste of scarce resources in medical research.

\section{Conclusions}

In our view, positive suggestions in any physician-patient relationship and peppermint pills for a sore throat are examples of important components of good doctoring. They should not be negatively labelled as some forms of placebo (“sham” or “dummy”) treatment.

Introduction of the new concept "impure placebo" to the side of the traditional "pure placebo", which has been universally called simply "placebo", leads to severe confusion. In particular, when "impure placebo" is pooled with "pure placebo" and together they are called a "placebo", readers are easily misled, since in the ordinary language "placebo" alone refers to "pure placebo". As we show in this paper, "impure placebo" is poorly defined. In addition, the examples given for impure placebos are unsound and in some cases absurd from the point of clinical work. "Impure placebo" is a useless concept and should not be used in scientific or medical literature. 


\section{References}

1. Howick, Jeremy, Felicity.L. Bishop, and Carl Heneghan, et al. 2013. Placebo use in the United Kingdom: results from a national survey of primary care practitioners. PLoS ONE 8(3):e58247.

http://dx.doi.org/10.1371/journal.pone.0058247

See comments in:

http://www.plosone.org/annotation/listThread.action?root=64407

https://helda.helsinki.fi/handle/10138/40220

2. Cheng, Maria. 2013. Many UK doctors give useless drugs, treatments. AP March 21.

http://www.newsvine.com/ news/2013/03/21/17396948-many-uk-doctors-give-useless-drugs-treatments. Accessed Dec 32014.

3. Philby, Charlotte. 2013. The placebo effect: doctors admit prescribing unproven treatments, unnecessary tests and pills with no active ingredient. The Independent, March 20, 2013.

http://www.independent.co.uk/life-style/health-and-families/health-news/the-placebo-effect-doctors-admit-pres cribing-unproven-treatments-unnecessary-tests-and-pills-with-no-active-ingredient-8542666.html. Accessed Dec 32014.

4. Grünbaum, Adolf. 1986. The placebo concept in medicine and psychiatry. Psychol Med 16:19-38. http://dx.doi.org/10.1017/S0033291700002506

5. Louhiala, Pekka, Raimo Puustinen. 2008. Rethinking the placebo effect. Medical Humanities 2008;34;107-109 http://dx.doi.org/10.1136/jmh.2008.000307

6. Moerman, Daniel E. 2002. Meaning, Medicine and the 'Placebo Effect'.

Cambridge: Cambridge University Press.

http://www.cambridge.org/es/academic/subjects/anthropology/social-and-cultural-anthropology/meaning-medic ine-and-placebo-effect

https://www.researchgate.net/publication/276090281 Meaning medicine and the placebo effect

http://www.directtextbook.com/isbn/9780521000871

http://dx.doi.org/10.1023/B\%3AMEDI.0000034422.21721.49

http://www.jstor.org/discover/10.2307/3655349

http://dx.doi.org/10.1525/maq.2003.17.4.501

7. Fässler, Margrit, Markus Gnadinger, Thomas Rosemann et al. 2009. Use of placebo interventions among Swiss primary care providers. BMC Health Serv Res 9:144.

http://dx.doi.org/10.1186/1472-6963-9-144

8. Meissner, Karin, Lisa Höfner, Margrit Fässler et al. 2012. Widespread use of pure and impure placebo interventions by GPs in Germany. Fam Pract 29:79-85.

http://dx.doi.org/10.1093/fampra/cmr045

9. Fässler, Margrit, Karin Meissner, Antonius Schneider et al. 2010. Frequency and circumstances of placebo use in clinical practice - a systematic review of empirical studies. BMC Med 8:15. http://dx.doi.org/10.1186/1741-7015-8-15

10. Gold, Harry, David B. Barr, McKeen Cattell et al. (editors). 1947. Cornell Conferences on Therapy: Use of Placebos in Therapy. New York: MacMillan, pp. 1-26.

11. Louhiala, Pekka. 2012. What do we really know about the deliberate use of placebos in clinical practice? $J$ Med Ethics 38:403-405.

http://www.ncbi.nlm.nih.gov/pubmed/22345556 http://dx.doi.org/10.1136/medethics-2011-100420

12. Gøtzsche, Peter. 1995. Placebo effects: concept of placebo should be discarded. BMJ 311:1640-1641. http://www.ncbi.nlm.nih.gov/pmc/articles/PMC2551527

13. Leslie, Alan. 1954. Ethics and practice of placebo therapy. Am J Med 16:854-862. http://dx.doi.org/10.1016/0002-9343(54)90450-7

14. Shapiro, Arthur, and Louis A. Morris. 1978. The placebo effect in medical and psychological therapies. In: Garfield, S.L. and A.E. Bergin (editors). Handbook and Psychotherapy and Behavior change: An Empirical Analysis. 2nd edition. New York: John Wiley \& Sons, pp. 369-410.

15. Hróbjartsson, Asbjörn. and Michael Norup. 2003. The use of placebo interventions in medical practice - a national questionnaire survey of Danish clinicians. Eval Health Prof 26:153-165. http://dx.doi.org/10.1177/0163278703026002002 
16. Fent, Rahel, Thomas Rosemann, Margrit Fässler et al. 2011. The use of pure and impure placebo interventions in primary care - a qualitative approach. BMC Family Practice 2011;12:11. http://dx.doi.org/10.1186/1471-2296-12-11

17. Kermen, Rachel, John Hickner, Howard Brody et al. 2010. Family physicians believe the placebo effect is therapeutic but often use real drugs as placebos. Fam Med 42:636-642. http://www.ncbi.nlm.nih.gov/pubmed/20927672

18. Altman, Douglas G. and J. Martin Bland. 1995. Absence of evidence is not evidence of absence. BMJ 311:485. http://dx.doi.org/10.1111/j.1751-0813.1996.tb13786.x

19. Kaiser, Laurent, Daniel Lew, Bernard Hirschel et al. 1996. Effects of antibiotic treatment in the subset of common-cold patients who have bacteria in nasopharyngeal secretions. Lancet 347:1507-1510. http://dx.doi.org/10.1016/S0140-6736(96)90670-4

20. Louhiala, Pekka.The ethics of the placebo in clinical practice revisited. J Med Ethics 2009;35:407-409. http://www.ncbi.nlm.nih.gov/pubmed/19567687 http://dx.doi.org/10.1136/jme.2008.028225

21. Young, Jim, An De Sutter, Dan Merenstein et al. 2008. Antibiotics for adults with clinically diagnosed acute rhinosinusitis: a meta-analysis of individual patient data. Lancet 371:908-914. http://dx.doi.org/10.1016/S0140-6736(08)60416-X

22. Linde, Klaus, Michael M. Berner and Levente Kriston. 2008. St. John's wort for major depression. Cochrane Database Syst Rev (4):CD000448. http://dx.doi.org/10.1002/14651858.CD000448.pub3

23. Novella, Steven. 2013. Do 97\% of UK Doctors Prescribe Placebos? NEUROLOGICA blog March28. http://theness.com/neurologicablog/index.php/do-97-of-uk-doctors-prescribe-placebos Accessed Dec 32014.

24. Holley Anthony D., E. Osland, J. Barnes et al. 2011. Scurvy: historically a plague of the sailor that remains a consideration in the modern intensive care unit. Intern Med J 41:283-285. http://dx.doi.org/10.1111/j.1445-5994.2010.02413.x

25. Raynaud-Simon, A., J. Cohen-Bittan, A. Gouronnec et al. 2010. Scurvy in hospitalized elderly patients. J Nutr Health Aging 14:407-410. http://dx.doi.org/10.1007/s12603-010-0032-y

26. Zhang, Michelle., Line Robitaille, Shaun Eintracht et al. 2011. Vitamin C provision improves mood in acutely hospitalized patients. Nutrition 27:530-533. http://dx.doi.org/10.1016/j.nut.2010.05.016

27. Wang, Yifan., Xing J. Liu, Line Robitaille et al. 2013. Effects of vitamin C and vitamin D administration on mood and distress in acutely hospitalized patients. Am J Clin Nutr 98:705-711. http://dx.doi.org/10.3945/ajcn.112.056366

28. Padayatty, Sebastian J., Hugh D. Riordan, Stephen M. Hewitt et al. 2006. Intravenously administered vitamin $\mathrm{C}$ as cancer therapy: three cases. CMAJ 174:937-942. http://dx.doi.org/10.1503/cmaj.050346

29. Hemilä, H. and Elizabeth Chalker. 2013. Vitamin C for preventing and treating the common cold. Cochrane Database Syst Rev (1):CD000980. http://dx.doi.org/10.1002/14651858.CD000980.pub4 http://www.mv.helsinki.fi/home/hemila/CC/CC.htm See a summary: http://www.ncbi.nlm.nih.gov/pmc/articles/PMC1160577

30. Hemilä, Harri. 2013. Vitamin C may alleviate exercise-induced bronchoconstriction: a meta-analysis. BMJ Open 3:e002416. http://dx.doi.org/10.1136/bmjopen-2012-002416 http://www.ncbi.nlm.nih.gov/pmc/articles/PMC3686214 See also: http://www.ncbi.nlm.nih.gov/pmc/articles/PMC4363347

31. Juraschek, Stephen P., Eliseo Guallar, Lawrence J. Appel et al. 2012. Effects of vitamin C supplementation on blood pressure: a meta-analysis of randomized controlled trials. Am J Clin Nutr 95:1079-1088. http://dx.doi.org/10.3945/ajcn.111.027995 